\title{
GEOCHEMISTRY OF PROTEROZOIC MAFIC ROCKS FROM THE PASSOS NAPPE (MINAS GERAIS, BRAZIL): TECTONIC IMPLICATIONS TO THE EVOLUTION OF THE SOUTHERN BRASILIA BELT
}

\author{
CLAUDIO DE MORISSON VALERIANO* \& LUIZSERGIO AMARANTE SIMÕES***
}

\begin{abstract}
RESUMO GEOQUÍMICA DAS ROCHAS MÁFICAS PROTEROZÓICAS DA NAPPE DE PASSOS (MINAS GERAIS, BRASIL): IMPLICATES TECTÔNICAS A EVOLUCAO DA FAIXA BRASÏLIA MERIDIONAL Os metabasitos intercalados nos metassedimentos do Grupo Araxá, no âmbito da nappe de Passos, porção meridional da Faixa Brasília, tem sua origem magmática investigada através da composição química, incluindo elementos terras raras (ETR). Os protólitos pré-metamórficos são de basaltos subalcalinos toléticos, pertencentes a três grupos composicionais: basaltos continentais de alto $>2 \%) \mathrm{TiO}_{2}$ (HTi), enriquecidos em $\mathrm{P} 2 \mathrm{Os}, \mathrm{Fe}_{2} \mathrm{O}_{3}^{\text {tot }}$, elementos incompatíveis e ETR leves (LaN/SmN entre 2,0 e 2,9); basaltos continentais de baixo $\mathrm{TiO}_{2}(\mathrm{LTi})$, relativamente aos anteriores menos enriquecidos em ETR leves ( $\mathrm{LaN} / \mathrm{SmN}$ entre 1,4 e 2,6); e basaltos muito semelhantes a E-MORB (LaN/SmN. entre 0.80 and 0.97$)$, volumetricamente subordinados. Os dois primeiros grupos são muito semelhantes aos tipos de alto e baixo $\mathrm{TiO}_{2}$ das províncias de platôs basálticos relacionados a fragmentação gondwânica (Paraná-Etendeka, Karroo). O terceiro representa contribuição magmática de fontes do Manto Empobrecido. Os corpos de HTi estao concentrados nas camadas inferiores da nappe de Passos, mas ocorrem em toda coluna estratigráfica; os de LTi ocorrem apenas nas porções inferiores da nappe; e os corpos de composição química do tipo E-MORB ocorrem apenas na parte superior. A distribuição dos metabasitos na coluna estratigráfica permite interpretar que durante a sedimentação das unidades que compõem a porção inferior da nappe a crosta continental estava em regime de distensão, que continuou ao longo de toda história sedimentar preservada. Durante a sedimentação das unidades superiores, 0 ambiente deposicional foi marinho relativamente profundo, e magmas do tipo MORB foram gerados, indicando um adelgaçamento litosférico mais severo. Entretanto, a persistência do magmatismo continental (HTi e LTi), neste estágio, sugere que a geração de assoalho oceânico não foi alcançada durante a distensão litosférica na porção meridional da Faixa Brasília.
\end{abstract}

Palavras-chaves: basaltos continentals, fontes mantélicas, Elementos Terras Raras, extensão litosférica, Orogênese Brasiliana

\begin{abstract}
Major and trace element compositions, including REE, are used to infer the tectonic setting of the mafic magmatism associated with the metasediments of the Araxá Group in the Passos nappe. The pre-metamorphic protoliths are sub-alkaline tholeiitic basalts, separated in three compositional groups: (i) high-TiOi (HTi) group (TiO2 $>2 \%$ ) enriched in $P_{2} \mathrm{O}_{5}, \mathrm{Fe}_{2} \mathrm{O}_{3}^{\text {tot }}$, incompatible elements, and light-REE (LaN/SmN between 2,0 and 2,9); (ii) low-TiOa (LTi), relatively depleted in these elements (LaN/SmN between 1,4 and 2,6); and (ii) a subordinated group represented by basalts of E-MORB composition, with LaN/SmN between 0.80 and 0.97 . The first two groups bear close similarities with the High and Low-TiOa magmas from Continental Flood Basalt provinces related to Gondwanaland break-up (Paraná-Etendeka, Karroo). The E-MORB like group represents magmatic contributions from depleted mantle sources. The HTi group samples are concentrated in the lower section, but occur along the whole stratigraphic column, whereas the LTi samples also occur in the lower portion of the nappe, and the MORB-like basalts were found only in the upper portion of the stratigraphic pile. The observed distribution of magma types favors the hypothesis of sin-depositional magmatism and indicates that the initial sedimentation in the Passos nappe metasedimentary pile (Lower Unit) was related to continental extension in association with CFB-type magmatism (HTi and LTi types), which continued along the rest of the preserved sedimentary record. During the sedimentation of the Upper Unit of the Passos nappe, in which relatively deeper marine conditions probably prevailed, MORB-like magmas were formed, probably related to more severe lithospheric extension and thinning. However, the persistence of continental magmatism in this stage is an indication that oceanic floor was not formed during lithospheric thinning in the southern segment of the Brasilia belt.
\end{abstract}

Keywords: Continental basalts; Mantle sources, Rare earth elements, Lithospheric extension, Brasiliano orogeny.

INTRODUCTION AND OBJECTIVE The Brasilia Belt is a major component of the Tocantins Province (Marini et al 1984), characterized by concentrated crustal convergence between the Amazon and São Francisco-Congo cratons (Figure la), during the Neoproterozoic Brasiliano orogeny (790-580 Ma.). It is thus a key piece in the paleocontinental puzzle before the assemblage of the Western Gondwana supercontinent. During the period between the Transamazonian (2.2-1.9 Ga.) and Brasiliano (790-580 Ma.) major erogenic events, reconstructions of continental fragmentation and drift in the Tocantins Province must seek, in the geological record, evidences to the questions of how much and where oceanic lithosphere was generated, and where extensional regimes achieved only continental rift systems. Moreover, the area of influence of Grenville-related orogenic processes (1.3-1.0 Ga.) still remains to be accurately mapped in central Brazil.

The former existence of oceanic lithosphere in the Tocantins province has been pointed out through the identification of petrotectonic assemblages, such as (i) ophiolitic thrust sheets in northern Araguaia belt (e.g. Teixeira 1996), between latitudes $0^{\circ}$ and $10^{\circ} \mathrm{S}$ (Figure la), (ii) in the Brasilia belt, an ophiolitic melange rock association within the Araxá Group, near the town of Abadiânia (Goiás), at latitude $16^{\circ} \mathrm{S}$ (Strieder \& Nilson 1992), and (iii) more to the west, Neoproterozoic (900-640 Ma.) calc-alkaline plutonic and volcanic com-

* Departamento de Geologia Regional e Geotectônica - Faculdade de Geologia - Universidade do Estado do Rio de Janeiro. Rua São Francisco Xavier n. 524/4006 - A Maracanã. CEP 20559-900 Rio de Janeiro - RJ - email: cmval@uerj.br

** Departamento de Petrologia e Metalogenia - Institute de Geociências e Ciências Exatas - Universidade Estadual Paulista - Caixa Postal 178, CEP 13506-900, Rio Claro, SP. 

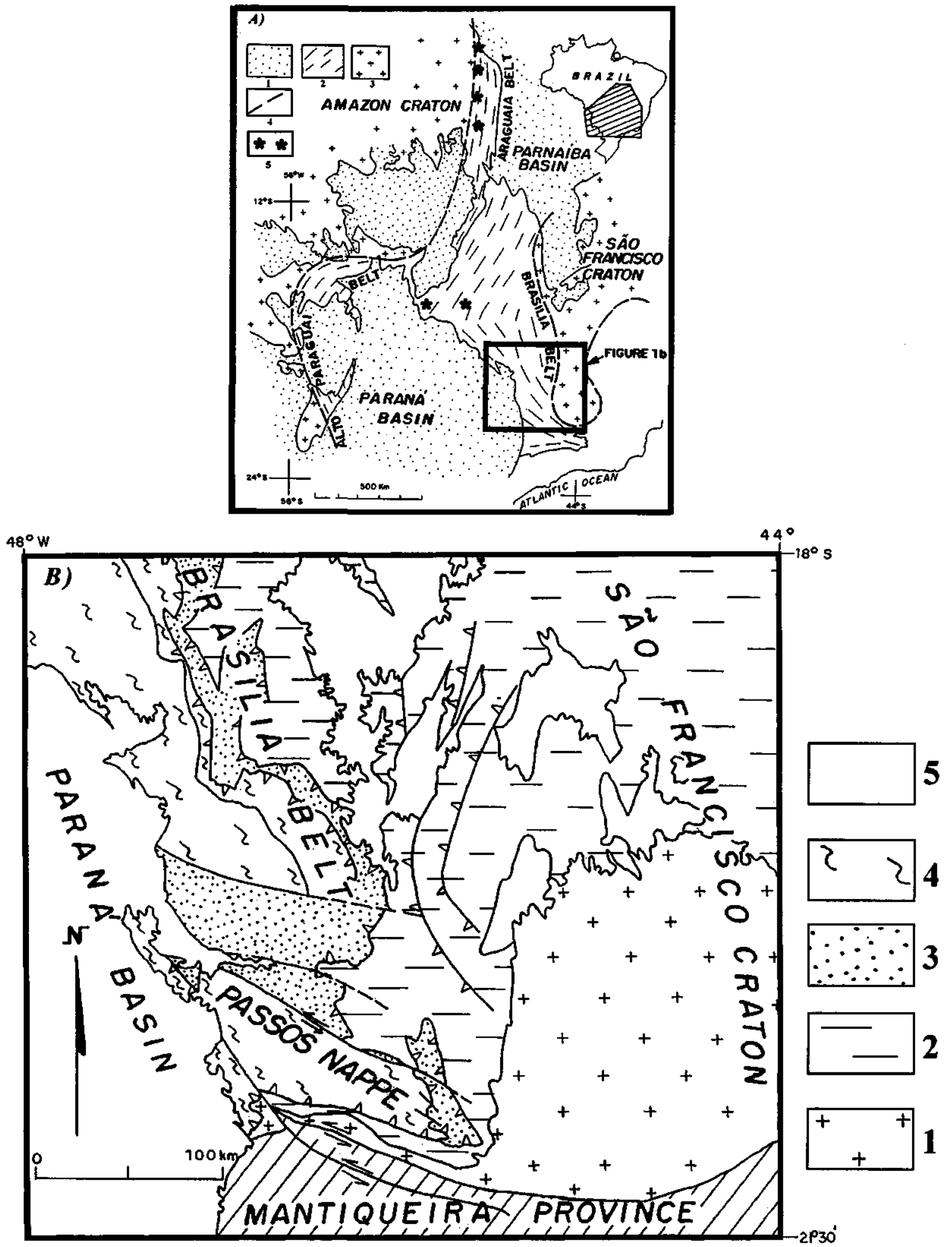

Figure 1 - (a) Tectonic sketch of the Tocantins Province between the Amazon and São Francisco cratons (simplified from Almeida et al. 1976). 1 -Phanerozoic cover; 2 - Tocantins Province; 3 -sin-Brasiliano cratons; 4 - cratonic boundaries; 5 -location of geological indications of the former existence of oceanic lithosphere. (b) Tectonic outline of the Southern Brasilia belt, with location of the Passos nappe (from Barbosa et al. 1970; Simdes \& Valeriano 1990; Szabo et al. 1993). 1 - cratonic basement; 2 cratonic cover (Bambui Group); 3 - External Allochthonous Domain; 4 -Internal Allochthonous Domain; 5 - Phanerozoic cover. Figura 1 - (a) Esboço tectônico da Província Tocantins (simplificado de Almeida et al. 1976). 1 -Cobertura fanerozóica; 2 - Província Tocantins; 3 - crátons sin-brasilianos; 4 - limites cratônicos; 5 - localização de indicadores geológicos da pretérita existência de litosfera oceânica. (b) Esboço tectônico da porção meridional da Faixa Brasília (compilado de Barbosa et al. 1970; Simões \& Valeriano 1990, Szabó et al. 1993). 1 - embasamento cratônico; 2 - cobertura cratônica (Grupo Bambuí); 3 -Domínio Alóctone Externo; 4 - Domínio Aló ctone Interne (incluindo a Nappe de Passos); 5 - cobertura fanerozóica. 
plexes, indicating B-subduction, were identified through isotope geochronology near Arenápolis (Goiás), at latitude $16^{\circ}$ 30'S (Pimentel \& Fuck 1992, 1994).

The geochemistry of mafic magmatism is a useful guide to the tectonic evolution of metasedimentary basins in which they commonly occur, especially in distinguishing compressional vs. extensional regimes, as well as continental vs. oceanic settings. This paper aims the lithogeochemical study of 20 selected samples of metabasic rocks which occur intercalated in the Araxá Group metasedimentary pile of the Passos nappe, southern Brasília belt (Figure 1b). Major and trace elements, including REE, are considered to constrain the former tectonic setting of the southernmost Araxá Group sedimentation.

\section{GEOLOGICAL CONTEXT OF THE MAFIC MAG-} MATISM The southern portion of the Brasilia belt is characterized by foreland thin-skinned thrusting and folding, with eastward directed tectonic transport of at least $150 \mathrm{~km}$ onto the southwestern border of the São Francisco craton, during the Brasiliano orogeny. Three main tectonic units are separated by major thrusts and display contrasting lithological, structural, geochronological and metamorphic characteristics (Simões \& Valeriano 1990, Valeriano 1992): the Autochthonous Domain is the continuation of the São Francisco craton, with deformed Neoproterozoic cover represented by the Bambuí Group; the External Allochthonous Domain is an imbricated thrust stack of low grade Proterozoic metasediments with Archean granite-greenstone association; and the Internal Allochthonous Domain (IAD), represented by the Passos nappe (Figure $1 \mathrm{~b}$ ), where metamoiphism and deformation are more intense.

The Passos nappe is the uppermost structural unit, composed of post-1.8 Ga. metasediments with thin lenses of greenschists and amphibolites of magmatic origin (probably dykes, sills and/or minor extrusions), which are the object of this paper. An inverted medium-P metamorphic gradient is conspicuous, ranging continuously from the biotite zone of greenschist facies, to upper amphibolite facies at the top (Simões 1995). The age of the metamorphic peak is poorly constrained in the Passos nappe. Post-metamorphic peak cooling is indicated by $\mathrm{K}-\mathrm{Ar}$ data on mineral separates (hornblende, biotite, muscovite) ranging from 674 to $566 \mathrm{Ma}$ (Correia et al 1982, Valeriano 1992).

Primary structures in the metasedimentary rocks have been pervasively obliterated by two generations of penetrative axial plane foliations to tight folds, besides late upright folding and vertical faulting. Field mapping of lithofacies in the Passos nappe (Teixeira \& Danni 1978, Schmidt 1983, Valeriano 1992, Simões 1995) defined a succession of 9 stratigraphic units, informally named from A to I (Figure 2). These were grouped in two contrasting sedimentary units in the Passos nappe, as previously proposed by Teixeira \& Danni (1978): the Lower Unit (ca. $700 \mathrm{~m}$ apparent thickness) comprises metapelites with carbonatic lenses and minor quartzite beds which, towards the top, become more frequent and thicker, suggesting a regressive shelf sequence, with increasing siliciclastics over carbonatic deposits; the Upper Unit (ca. $3000 \mathrm{~m}$ apparent thickness) initiates with paragneisses displaying gradational contact with the underlying mica schists with minor fine grained quartzites of the top of the Lower Unit. This transition is also a stratigraphic horizon of concentrated occurrence of metabasic rocks, marking the onset of

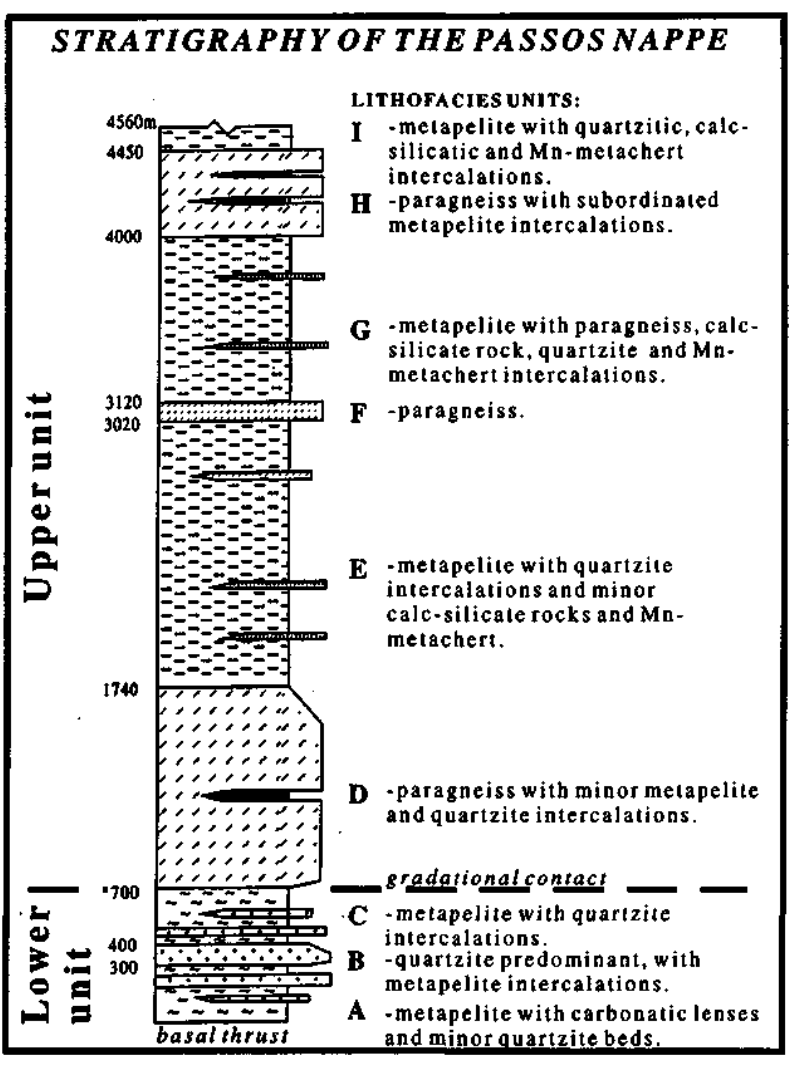

Figure 2 - Representative stratigraphic column of the Passos nappe (Simões 1995).

Figura 2 - Coluna estratigráfica representativa da Nappe de Passos (Simões 1995).

tectonically more unstable sedimentation conditions, in which erosion of granite-gneissic basement and/or other sources led to the abundant paragneisses and feldspathic schists containing quartz-feldspar clasts. Towards the top, the Upper Unit displays predominant metapelites with minor paragneiss and thin beds of calc-silicate rocks and manganesiferous metachert. Also associated with more frequent intercalations of metabasic rocks, this association of lithofacies points to a probably deeper marine environment.

METABASIC ROCKS Occurrence and petrography The metabasic rocks occur along the whole preserved metasedimentary succession as boudins and disrupted lenses parallel to the main foliation of the host rocks. They display the same metamorphic and polydeformational history of the metasediments, indicating an origin related to either sin-sedimentary extrusion or dyke/sill emplacement, before tectonic inversion of the basin. However, their systematic compositional variation in relation to the stratigraphic position, as discussed below, suggests extrusive processes during sedimentation. In general, the Upper Unit is more abundant in mafic intercalations. The top of the Lower Unit is also especially rich in mafic intercalations, possibly marking an extensional pulse that started the sedimentation of the Upper Unit.

The mineralogy of the metabasic rocks is chiefly controlled by metamorphic grade, without major influence of the chemical compositional variation on the petrographic charac- 
Table 1 - Geographic and stratigraphic location of analysed samples, with brief petrographic description Tabela 1 - Localização geográ fica e estratigrá fica das amostras analisadas e breve descrição petrográ fica

\begin{tabular}{|c|c|c|c|c|c|}
\hline Sample & $\begin{array}{l}\text { UTM } \\
\text { coordinates }\end{array}$ & Unit & $\begin{array}{l}\text { Metamorphic } \\
\text { facies }\end{array}$ & Lithology & $\begin{array}{l}\text { Mineralogy in decreasing order } \\
\text { (accessory minerals) }\end{array}$ \\
\hline LN-5b & $303.00-7737.50$ & $\mathbf{H}$ & amphibolite & homogeneous amphibolite & hbl-ep-plag-qz-bi (sph-ilm-mag) \\
\hline CA-4-96 & $300.50-7734.10$ & $\mathbf{H}$ & amphibolite & $\begin{array}{l}\text { amphibolite with plagioclase rimmed gamet } \\
\text { aggregates }\end{array}$ & hbl-plag-gar-qz-sph \\
\hline LDS-56b & $298.10-7743.80$ & $\mathbf{E}$ & amphibolite & homogeneous amphibolite & hbl-ep-plag-qz (sph-ap-ilm) \\
\hline DEL-1-89k & $295.60-7747.10$ & $\mathrm{E}$ & amphibolite & homogeneous amphibolite & hbl-ep-zo-plag-qz-ru (sph-ap-op) \\
\hline DEL-1-89h & $295.60-7747.10$ & $\mathbf{E}$ & amphibolite & garnet amphibolite & hbl-ep-plag-qz-gar-sph-act-ru (ilm-bi) \\
\hline DEL-1-61 & $299.30-7750.70$ & $\mathbf{E}$ & amphibolite & homogeneous amphibolite & hbl-ep-qz-plag (sph-ilm-mag-act) \\
\hline ZS-71 & $384.55-7690.25$ & D & upper greenschist & $\begin{array}{l}\text { fine grained chlorite schist with magnetite } \\
\text { porphiroblasts }\end{array}$ & chl-bi-alb-ep-zo-clzo-qz (ilm-ap-sph) \\
\hline CRC-4-8 & $386.95-7689.65$ & $\mathrm{D}$ & upper greenschist & fine grained homogeneous chlorite schist & hbl-chl-alb-ep-zo-clzo-qz (ilm-ap-sph) \\
\hline CRC-4-34 & $386.35-7689.65$ & D & upper greenschist & fine grained homogeneous chlorite schist & hbl-chl-alb-ep-zo-clzo-qz (ilm-sph-ap) \\
\hline CRC-4-35 & $385.87-7689.90$ & $\mathrm{D}$ & upper greenschist & fine grained homogeneous chlorite schist & chl-alb-hbl-ep (sph-op-ap) \\
\hline AL-MA-54 & $351.15-7693.35$ & D & upper greenschist & biotite-bearing chlorite schist & bi-chl-alb-qz-op (ep-zo-clzo-ap-tour) \\
\hline $1-59 \mathrm{~d}$ & $315.30-7709.25$ & $\mathrm{C}$ & upper greenschist & chlorite-amphibole schist & hbl-plag-op-ep-bi (sph-ap-zo) \\
\hline $\mathrm{I}-120 \mathrm{~b}$ & $312.90-7707.85$ & $\mathrm{C}$ & upper greenschist & chlorite-amphibole schist & hbl-plag-chl-ep-zo-qz-op (ap-sph) \\
\hline $\mathbf{I}-119 \mathrm{~b}$ & $312.70-7707.85$ & $\mathrm{C}$ & upper greenschist & amphibole schist with retrogressive biotite & plag-hbl-bi-qz-wp-mag-carb (ap-sph) \\
\hline SJB-3-43 & $339,00-7751.00$ & A & greenschist & $\begin{array}{l}\text { fine grained chlorite schist with } \\
\text { quartz-feldspathic lenses }\end{array}$ & alb-ep-chl-qz-mag-ilm (sph-bi) \\
\hline SJB-3-55b & $337.75-7752.50$ & A & greenschist & fine grained chlorite schist & alb-chl-ep-mag-ilm (sph-bi-wm) \\
\hline SJB-3-55d & $337.75-7752.50$ & A & greenschist & $\begin{array}{l}\text { fine grained chlorite schist with albite } \\
\text { porphiroblasts }\end{array}$ & alb-ep-chl-qz-mag-ilm-wm(sph) \\
\hline IL-4-41b & $421.20-7677.10$ & A & greenschist & fine grained chlorite schist & chl-alb-ep-op-act (ap-tour-carb) \\
\hline RP-2-52 & $283.70-7757.80$ & A & greenschist & fine grained chlorite schist & alb-ep-chl-act-mag-qz (sph) \\
\hline RP-3-134 & $283.50-7759.10$ & $\mathbf{A}$ & greenschist & fine grained actinolite schist & alb-ep-act-chl-qz \\
\hline
\end{tabular}

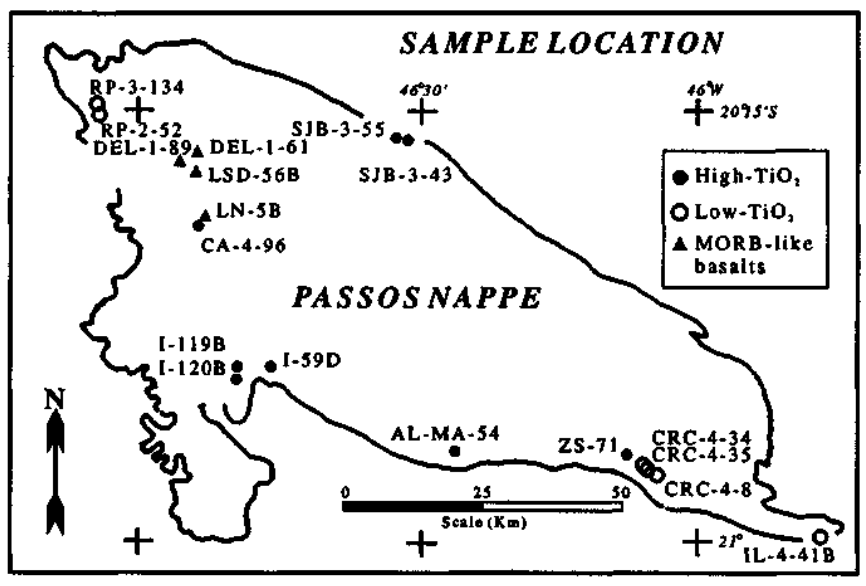

Figure 3 - Location of the analysed samples in the Passes nappe.

Figura 3 - Localização das amostras analisadas na Nappe de Passos.

teristics. A brief description of the metamorphic mineralogy of the analysed samples is presented in Table 1.

\section{Sampling, analytical procedures and results}

The map location of the samples is shown in Figure 3, with UTM coordinates in Table 1. Analyses were performed at Geosol-Geolab Division Laboratory, Brazil, through the following methods: $\mathrm{Si}, \mathrm{Al}, \mathrm{Ti}, \mathrm{Ca}, \mathrm{Fe}^{\text {tot }}, \mathrm{P}$ and trace elements were measured by X-ray fluorescence spectrophotometer;
$\mathrm{Mn}, \mathrm{Ca} . \mathrm{Na}, \mathrm{K}$ and $\mathrm{Mg}$ by atomic absorption spectrophotometer; $\mathrm{Fe}^{2+}$ by wet chemistry procedures; REE contents were measured by fusion-ICP. Major and trace element compositions are shown in Tables 2 to 4.

Chemical alteration effects Possible processes which alter the original chemical composition of the mafic rocks from the Passes nappe may include: water interaction during magma crystallization; hydrothermal alteration; and water income during progressive low grade metamorphism. For the samples which attained amphibolite facies metamorphism (see Table 1), further dehydration must have occurred. Chemical modifications were detected in a few samples, through the binary correlation diagrams (Fig 5a, b) and by criteria of Miyashiro (1975) and Mullen (1983). Sample ALMA-54 has anomalous composition and, with relation to $\mathrm{MgO}$, displays significantly high contents in $\mathrm{Na}_{2} \mathrm{O}, \mathrm{K}_{2} \mathrm{O}$, $\mathrm{P}_{2} \mathrm{O}_{5}, \mathrm{Zr}, \mathrm{Rb}$, and $\mathrm{Nb}$, with low $\mathrm{CaO}$ content. Samples I-59d and $\mathrm{I}-119 \mathrm{~b}$ show enrichment in $\mathrm{NaaO}$ and $\mathrm{K}_{2} \mathrm{O}$, and $\mathrm{Rb}$ mobilization. Depletion in $\mathrm{Ba}, \mathrm{Sr}$ (CA-4-96) and Ce (CA-496, CRC-4-8) were also detected. Nevertheless, these effects are restricted to a few samples and acted selectively on the mobile elements $\left(\mathrm{Na}_{2} \mathrm{O}, \mathrm{K}_{2} \mathrm{O}, \mathrm{Rb} \mathrm{Ba}, \mathrm{Sr}\right)$, not significantly modifying the relatively immobile elements, especially $\mathrm{TiO}_{2}$, $\mathrm{P}_{2} \mathrm{O}_{5}, \mathrm{Zr}, \mathrm{Nb}, \mathrm{Y}$ and the REE. Therefore, as discussed below, the identification of compositional groups and inferences on the tectonic setting of the mafic magmatism are focused on the latter elements, which display fairly consistent trends in the binary correlation (Figure 5), normalized (Figures 6,7) and tectonic discrimination diagrams (Figure 8). 
Table 2 - Major, trace and rare earth element contents of the high-TiO ${ }_{2}(>2 \%)$ metabasic rocks from the Passos nappe, with anhydrous average and standard deviaton $(\delta \mathrm{n})$.

Tabela 2 - Teores de elementos maiores, traços e terras raras das rochas metabásicas de alto $\mathrm{TiO}_{2}(>2 \%)$ da nappe de Passes, com média anidra e desvio padrão (סn).

\begin{tabular}{|c|c|c|c|c|c|c|c|c|c|c|c|c|}
\hline Sample & 1+59d & I-119b & I-120b & ZS-71 & $\begin{array}{c}\text { SJB-3- } \\
\mathbf{5 5 b}\end{array}$ & $\begin{array}{c}\text { SJBB-3- } \\
\mathbf{5 5 d}\end{array}$ & $\begin{array}{c}\text { SJB-3- } \\
43\end{array}$ & $\underset{96}{C A}+$ & $\begin{array}{c}\text { LSD- } \\
\mathbf{5 6 b}\end{array}$ & $\underset{54}{\text { AL-MA- }}$ & $\begin{array}{c}\text { Anhydrous } \\
\text { average }\end{array}$ & $\delta_{n}$ \\
\hline $\mathrm{SiO}_{2}$ & 54.0 & 49.6 & 51.8 & 50.5 & 51.5 & 47.7 & 49.1 & 51.7 & 48.6 & 49.0 & 51.7 & 1.5 \\
\hline $\mathrm{TiO}_{2}$ & 2.9 & 3.3 & 2.9 & 3.1 & 3.2 & 3.2 & 2.2 & 3.1 & 3.4 & 3.4 & 3.2 & 0.4 \\
\hline $\mathrm{Al}_{2} \mathrm{O}_{3}$ & 12.5 & 13.3 & 14.2 & 13.8 & 13.9 & 16.7 & 14.4 & 12.5 & 14.0 & 13.4 & 14.3 & 1.1 \\
\hline $\mathrm{Fe}_{2} \mathrm{O}_{3}$ & 7.9 & 7.1 & 6.0 & 8.9 & 9.3 & 7.8 & 9.2 & 2.9 & 5.3 & 5.7 & 7.2 & 2.0 \\
\hline $\mathrm{FeO}$ & 7.0 & 7.7 & 7.7 & 6.5 & 4.2 & 6.3 & 5.6 & 12.2 & 10.5 & 9.0 & 7.9 & 2.3 \\
\hline Mno & 0.17 & 0.25 & 0.25 & 0.20 & 0.18 & 0.18 & 0.20 & 0.29 & 0.28 & 0,13 & 0.22 & 0.05 \\
\hline $\mathrm{MgO}$ & 2.7 & 3.3 & 3.4 & 3.5 & 3.7 & 3.9 & 4.6 & 4.7 & 5.0 & 6.1 & 4.2 & 1.0 \\
\hline $\mathrm{CaO}$ & 5.2 & 5.4 & 7.0 & 7.0 & 5.8 & 5.5 & 6.1 & 9.0 & 9.0 & 2.6 & 6.4 & 1.8 \\
\hline $\mathrm{Na}_{2} \mathrm{O}$ & 5.4 & 4.6 & 3.3 & 2.0 & 3.4 & 2.7 & 3.0 & 1.9 & 0.78 & 4.5 & 3.2 & 1.4 \\
\hline $\mathrm{K}_{2} \mathrm{O}$ & 0.29 & 2.50 & 0.86 & 1.10 & 1.00 & 1.30 & 1.80 & 0.28 & 0.28 & 1.80 & 1.16 & 0.73 \\
\hline $\mathrm{P}_{2} \mathrm{O}_{5}$ & 0.59 & 0.66 & 0.55 & 0.72 & 0.65 & 0.53 & 0.27 & 0.17 & 0.48 & 0.81 & 0.56 & 0.19 \\
\hline $\mathrm{H}_{2} \mathrm{O}$ & 0.99 & 0.98 & 1.43 & - & 2.68 & 3.81 & 2.73 & 0.70 & 1.16 & 2.99 & & \\
\hline $\mathrm{CO}_{2}$ & 0.25 & 1.00 & 0.40 & - & 0.25 & 0.25 & 0.55 & 0.25 & 1.00 & 0.40 & & \\
\hline LOI & 0.15 & 0.16 & 0.15 & 1.88 & 0.24 & 0.06 & 0.12 & 0.32 & 0.16 & 0.57 & & \\
\hline $\begin{array}{l}\text { Total } \\
\text { ppm: }\end{array}$ & 100.04 & 99.85 & 99.94 & 99.20 & 100.00 & 99.93 & 99.87 & 100.01 & 99.94 & 100.40 & 100.04 & \\
\hline $\mathrm{Cr}$ & 68 & 68 & 68 & 34 & 68 & 68 & 68 & 68 & 68 & 36 & 63 & 14 \\
\hline $\mathrm{Ni}$ & 79 & 79 & 79 & 27 & 79 & 79 & 79 & 79 & 79 & 59 & 74 & 16 \\
\hline Co & 62 & 72 & 77 & 47 & 67 & 77 & 82 & 87 & 82 & - & 74 & 12 \\
\hline$v$ & 160 & 170 & 160 & 190 & 170 & 190 & 220 & 470 & 320 & + & 233 & 99 \\
\hline $\mathbf{R b}$ & 8 & 42 & 19 & 23 & 21 & 24 & 26 & 8 & 10 & 27 & 21 & 10 \\
\hline $\mathbf{B a}$ & 81 & 1164 & 672 & 520 & 985 & 564 & 546 & 45 & 54 & 1270 & 608 & 438 \\
\hline Sr & 130 & 170 & 280 & 320 & 250 & 510 & 200 & 75 & 68 & 75 & 214 & 137 \\
\hline $\mathrm{Nb}$ & 24 & 27 & 24 & 23 & 27 & 22 & 17 & 18 & 42 & 26 & 26 & 7 \\
\hline $\mathbf{z r}$ & 300 & 340 & 290 & 230 & 320 & 280 & 210 & 190 & 190 & 230 & 265 & 53 \\
\hline $\mathbf{Y}$ & 100 & 45 & 41 & 42 & 41 & 55 & 35 & 52 & 32 & 31 & 49 & 19 \\
\hline $\mathrm{Cl}$ & 78 & 120 & 84 & - & $<20$ & $<20$ & 26 & 88 & 250 & $<20$ & & \\
\hline $\mathrm{La}$ & 56.57 & 36.39 & 32.09 & 45.21 & 32.38 & 26.12 & 22.62 & 11.29 & 21.22 & 46.41 & 33.92 & 13.14 \\
\hline $\mathrm{Ce}$ & 51.91 & 85.36 & 71.86 & 100.30 & 71.94 & 59.90 & 55.67 & 26.52 & 50.90 & 98.74 & 69.23 & 22.71 \\
\hline Nd & 59.02 & 44.40 & 37.45 & 54.70 & 37.23 & 34.16 & 28.41 & 15.49 & 27.17 & 53.59 & 40.24 & 13.42 \\
\hline Sm & 12.13 & 9.13 & 7.87 & 11.27 & 7.72 & 7.43 & 5.98 & 3.50 & 6.05 & 10.95 & 8.43 & 2.63 \\
\hline Eu & 2.63 & 1.90 & 1.74 & 2.41 & 1.75 & 1.86 & 1.40 & 0.82 & 1.74 & 2.19 & 1.90 & 0.50 \\
\hline Gd & 12.30 & 7.67 & 6.70 & 9.37 & 6.59 & 7.40 & 5.42 & 3.20 & 5.39 & 9.22 & 7.53 & 2.45 \\
\hline Dy & 11.56 & 6.96 & 6.07 & 9.44 & 5.88 & 7.14 & 5.15 & 2.93 & 4.69 & 9.32 & 7.10 & 2.49 \\
\hline Ho & 2.40 & 1,43 & 1.24 & 1.85 & 1.20 & 1.49 & 1.08 & 0.63 & 0.96 & 1.88 & 1.45 & 0.50 \\
\hline $\mathbf{E r}$ & 6.93 & 4.03 & 3.43 & 4.75 & 3.38 & 4.34 & 3.12 & 1.91 & 2.67 & 5.13 & 4.01 & 1.37 \\
\hline $\mathbf{Y b}$ & 4.93 & 3.24 & 2.75 & 3.37 & 2.61 & 3.69 & 2.32 & 1.59 & 2,15 & 3.72 & 3.12 & 0.93 \\
\hline Lu & 0.59 & 0.41 & 0.34 & 0.36 & 0.30 & 0.48 & 0.27 & 0.20 & 0.28 & 0.42 & 0.38 & 0.11 \\
\hline Mg\# & 25.43 & 29.45 & 31.63 & 30.06 & 34.41 & 34.29 & 37.13 & 36.12 & 36.85 & 43.48 & 33.89 & 4.78 \\
\hline
\end{tabular}


Table 3 - Major, trace and rare-earth element contents of the low-TiO ${ }_{2}(<2 \%)$ metabasic rocks from the Passos nappe, with anhydrous average and standard deviaton ( $\delta \mathrm{n})$.

Tabela 3 - Teores de elementos maiores, traços e terras raras das rochas metabásicas de baixo $\mathrm{TiO}_{2}(<2 \%)$ da nappe de Passos, com média anidra e desvio padrão

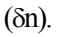

\begin{tabular}{|c|c|c|c|c|c|c|c|c|}
\hline Sample & IL-4-41b & CRC-4-34 & CRC-4-35 & RP-3-134 & RP-2-52 & CRC-4-8 & $\begin{array}{l}\text { Anhydrous } \\
\text { average }\end{array}$ & $\delta_{\mathrm{n}}$ \\
\hline $\mathrm{SiO}_{2}$ & 49.8 & 48.8 & 48.4 & 45.7 & 49.3 & 46.9 & 49.8 & 1.6 \\
\hline $\mathrm{TiO}_{2}$ & 1.7 & 1.5 & 1.2 & 1.4 & 1.2 & 1.3 & 1.4 & 0.2 \\
\hline $\mathrm{Al}_{2} \mathrm{O}_{3}$ & 14.7 & 16.0 & 16.5 & 16.0 & 15.5 & 17.4 & 16.6 & 0.8 \\
\hline $\mathrm{Fe}_{2} \mathrm{O}_{3}$ & 6.0 & 5.1 & 4.6 & 8.2 & 5.8 & 5.3 & 6.0 & 1.2 \\
\hline $\mathrm{FeO}$ & 7.0 & 7.4 & 6.8 & 5.6 & 4.9 & 6.7 & 6.6 & 0.9 \\
\hline $\mathrm{MnO}$ & 0.23 & 0.23 & 0.21 & 0.22 & 0.16 & 0.21 & 0.22 & 0.02 \\
\hline $\mathrm{MgO}$ & 5.8 & 6.3 & 7.4 & 6.7 & 7.3 & 7.5 & 7.1 & 0.6 \\
\hline $\mathrm{CaO}$ & 5.8 & 9.0 & 7.3 & 9.7 & 9.1 & 8.6 & 8.5 & 1.3 \\
\hline $\mathrm{Na}_{2} \mathrm{O}$ & 3.1 & 3.0 & 3.8 & 2.6 & 2.5 & 2.5 & 3.0 & 0.5 \\
\hline $\mathrm{K}_{2} \mathrm{O}$ & 1.40 & 0.26 & 0.65 & 0.41 & 0.70 & 0.27 & 0.64 & 0.41 \\
\hline $\mathrm{P}_{2} \mathrm{O}_{5}$ & 0.28 & 0.20 & 0.13 & 0.16 & 0.09 & 0.13 & 0.17 & 0.06 \\
\hline $\mathrm{H}_{2} \mathrm{O}$ & 3.24 & 1.73 & 2.65 & 2.85 & 2.99 & 3.23 & & \\
\hline $\mathrm{CO}_{2}$ & 0.70 & 0.25 & 0.25 & 0.25 & 0.25 & 0.25 & & \\
\hline LOI & 0.19 & 0.46 & 0.51 & 0.12 & 0.07 & 0.46 & & \\
\hline $\begin{array}{l}\text { Total } \\
\text { ppm: }\end{array}$ & 99.94 & 100.23 & 100.40 & 99.91 & 99.86 & 100.75 & 100.03 & \\
\hline $\mathrm{Cr}$ & 68 & 200 & 230 & 137 & 205 & 220 & 182 & 58 \\
\hline $\mathrm{Ni}$ & 157 & 120 & 160 & 236 & 157 & 170 & 173 & 36 \\
\hline Co & 82 & $=$ & $=$ & 103 & 72 & - & 89 & 13 \\
\hline v & 220 & - & - & 260 & 240 & . $\quad-$ & 249 & 16 \\
\hline $\mathbf{R b}$ & 30 & 12 & 18 & 11 & 20 & 15 & 18 & 7 \\
\hline $\mathrm{Ba}$ & 528 & 58 & 200 & 206 & 358 & 68 & 245 & 172 \\
\hline $\mathrm{Sr}$ & 270 & 260 & 220 & 220 & 250 & 270 & 257 & 22 \\
\hline $\mathbf{N b}$ & 17 & $<10$ & $<10$ & 12 & 13 & $<10$ & $<12$ & \\
\hline $\mathbf{Z r}$ & 200 & 98 & 72 & 110 & 110 & 75 & 115 & 45 \\
\hline $\mathbf{Y}$ & 28 & 10 & 10 & 26 & 24 & 23 & 21 & 8 \\
\hline $\mathbf{a}$ & $<20$ & $<20$ & $<20$ & 34 & 53 & $<20$ & $<28$ & \\
\hline La & 21.53 & 11.28 & 8.42 & 13.91 & 9.41 & 27.62 & 15.89 & 7.23 \\
\hline $\mathrm{Ce}$ & 46.45 & 30.23 & 21.45 & 33.21 & 22.76 & 22.98 & 30.53 & 9.15 \\
\hline Nd & 24.78 & 16.22 & 12.62 & 23.00 & 14.94 & 34.83 & 21.78 & 8.05 \\
\hline Sm & 5.20 & 3.90 & 3.02 & 6.19 & 3.54 & 8.08 & 5.16 & 1.81 \\
\hline Eu & 1.23 & 1.06 & 0.94 & 1.58 & 0.95 & 2.32 & 1.39 & 0.51 \\
\hline Gd & 4.59 & 3.60 & 2.95 & 6.72 & 3.48 & 7.49 & 4.97 & 1.77 \\
\hline Dy & 4.06 & 4.04 & 3.59 & 7.81 & 3.56 & 8.12 & 5.37 & 2.04 \\
\hline Ho & 0.83 & 0.84 & 0.66 & 1.63 & 0.77 & 1.55 & 1.08 & 0.40 \\
\hline $\mathrm{Er}$ & 2.33 & 2.43 & 2.04 & 4.69 & 2.37 & 3.83 & 3.05 & 1.00 \\
\hline $\mathrm{Yb}$ & 1.66 & 2.07 & 1.62 & 4.20 & 2.05 & 2.94 & 2.51 & 0.94 \\
\hline Lu & 0.18 & 0.26 & 0.19 & 0.56 & 0.26 & 0.33 & 0.31 & 0.13 \\
\hline Mg\# & 45.46 & 48.36 & 54.66 & 47.91 & 56.25 & 53.82 & 52.58 & 6.54 \\
\hline
\end{tabular}



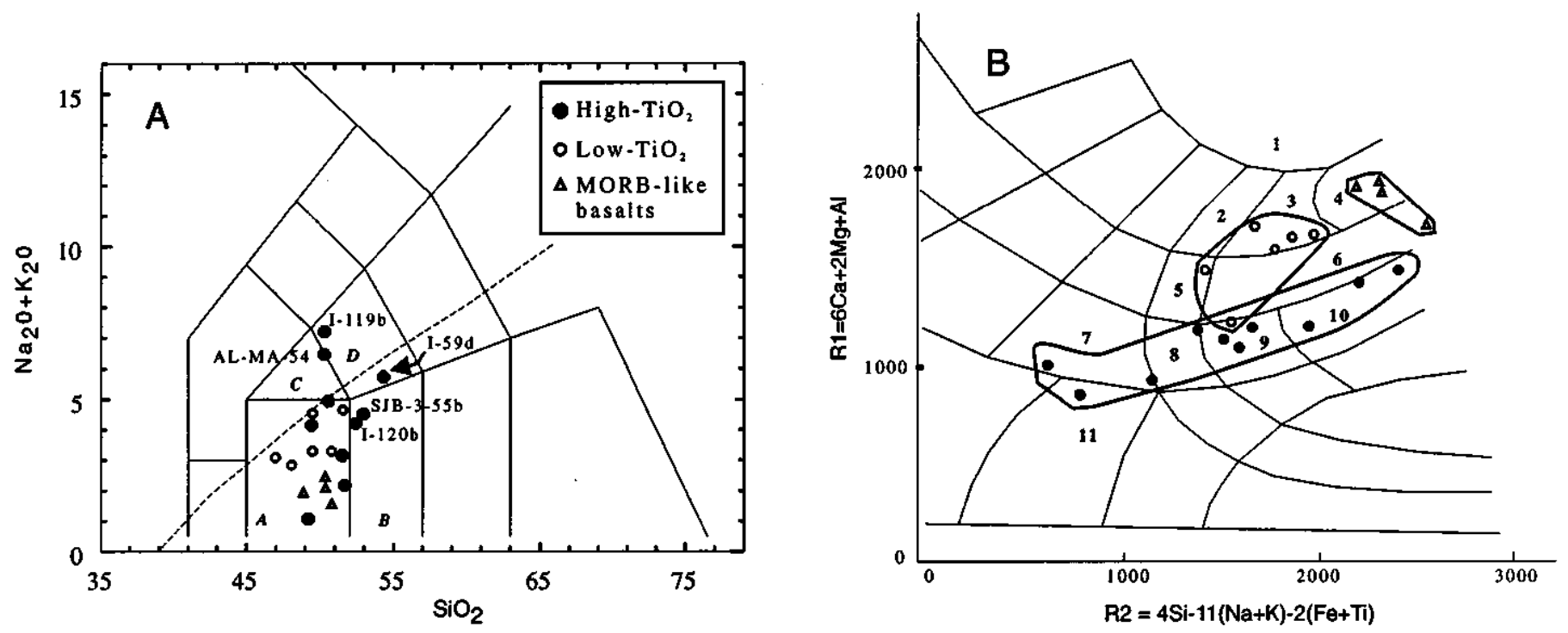

Figure 4 - Chemical classification of metabasic rocks, (a) Le Bas et al. (1986). Compositional fields: A -basalt; B - basaltic andesite; $C$ - trachy-basalt; D -basaltic trachy-andesite. Dashed line indicates the alkaline-subalkaline boundary of Irvine \& Baragar (1971); (b) De la Roche et al. (1980). Compositional fields: 1 - picrite; 2 - alkali-basalt; 3 - olivine basalt; 4 -Tholeiite; 5 - lati-basalt; 6 - andesi-basalt; 7 -trachy-andesite; 8 - latite; 9 - lati-andesite; 10 -andesite; 11 - trachyte.

Figura 4 - Classificação química das rochas metabásicas.(a) Le Bas et al. (1986). Campos composicionais: A - basalto; B - andesito basáltico; C -traquibasalto; D - traquiandesito basáltico. A linha pontilhada separa os campos de rochas alcalinas e subalcalinas de Irvine \& Baragar (1971);(b) De la Roche et al. (1980). Campos composicionais: 1 - picrito; 2 -alcali-basalto; 3 - olivinabasalto; 4 - toleito; 5 -lati-basalto; 6 - andesi-basalto; 7 - traqui-andesito; 8 -latito; 9 - lati-andesito; 10 - andesito; 11 - traquito.

Magma types and geochemical characteristics On the basis of the Total alkalis x Silica diagram (Figure 4a), the studied samples are classified as subalkaline tholeiitic basalts (15) and basaltic andesites (2), with $\mathrm{SiO}_{2}$ ranging from 47 to $52 \mathrm{wt} \%$, except for 3 basaltic trachyandesites, two of which are alkaline. An alternative classification, the R1R2 diagram (De la Roche et al. 1980), is given in Figure $4 \mathrm{~b}$. The $\mathrm{MgO}$ contents (Figure 5) range from 3 to 9 $\mathrm{wt} \%$, with a positive correlation with $\mathrm{CaO}$, indicating a major compositional control of plagioclase-clinopyroxene fractional crystallization.

On the basis of major and trace element trends (Figure 5), including REE (Figure 6), three compositional groups are identified: high- $\mathrm{TiO}_{2}$ metabasalts $(\mathrm{HTi})$, low- $\mathrm{TiO}_{2}$ metabasalts (LTi), and a third group represented by subordinated MORB-like basalts (MLB). After a brief description of their general compositional characteristics, the tectonic characterization and the implications of their stratigraphic distribution are discussed below.

HIGH-TIO2 MAFIC ROCKS (HTI) The HTi group (10 samples) represents relatively the most evolved magmas, with $\mathrm{Mg}$ \# between 25 and 44 (Table 2), and marked enrichment in Ti, Fe, $\mathrm{P}$ and also in $\mathrm{Nb}, \mathrm{Zr}, \mathrm{Y}$ and Ba. The chondritenormalized REE patterns (Figure 6a) are the most fractionated, especially the light-REE, with $\mathrm{LaN} / \mathrm{SmN}$ ranging from 2.0 to 2.9. A conspicuous negative $\mathrm{Eu}$ anomaly is observed (average $\mathrm{Eu} / \mathrm{Eu}^{*}=0.72$ ). Low $\mathrm{Mg \#}$ (below 44) and Ni contents below $79 \mathrm{ppm}$ (Table 2), and a positive correlation of $\mathrm{Al}_{2} \mathrm{O}_{3}$ and $\mathrm{CaO}$ with $\mathrm{MgO}$ (Figure 5) indicate respectively high rates of initial olivine fractionation, and plagioclase+clinopyroxene fractional crystallization.

The REE pattern abundances and the MORB-normalized multielemental diagram of Figure 7a point to the close similarity of the HTi metabasalt compositions to basalts from classical continental flood basalt (CFB) provinces (Cox 1978), such as the Jurassic Serra Geral formation, Parana Basin (Bellieni et al. 1986), and the Recent Snake River plain volcanics (Thompson et al. 1983), characteristically enriched in LIL elements, with respect to MORE.

In the major element tectonic discrimination diagram of Pearce et al. (1977) the sub-alkcaline samples with $\mathrm{SiO}_{2}>$ $51 \%$ plot in the continental field (Figure 8a). In the trace element diagrams of Figure $8 \mathrm{~b}$ to $8 \mathrm{~d}$, these samples plot preferentially in the intraplate or MORE fields, a characteristic displayed also by the classical examples of continental mafic magmatism cited in the previous paragraph.

\section{LOW-TiO ${ }_{2}$ MAFIC ROCKS (Ti)}

The LTi group $(6$ samples), in relation to the $\mathrm{HTi}$, represents more primitive magmas (Mg\# 45-57, see Table 2), depleted in incompatible elements, and with less fractionation of light-REE ( $\mathrm{LaN} / \mathrm{SmN}$ between 1.4 and 2.6). A less pronounced negative Eu anomaly is also observed (average $\mathrm{Eu} / \mathrm{Eu}^{*}=0.84$ ).

Figure $7 \mathrm{~b}$ shows that the LTi metabasalts have similar compositions to other continental tholeiitic basalts from the Paleoproterozoic (2.1-1.8 Ga.) mafic NW and ENE trending dyke swarms of the Superior Province, Canada (Condie et al. 1987) and from the Cretaceous-Tertiary Deccan plateau, India (Thompson et al. 1983), for example. As the HTi, the LTi group samples scatter in and around the intraplate and MORE fields of the trace element tectonic discrimination diagrams of Figure 8.

MORB-LIKE METABASALTS This group (4 samples) represents the most primitive magmas (Mg\# between 46 and 61, see Table 4), with higher $\mathrm{Mg}, \mathrm{Ca}, \mathrm{Cr}$ and $\mathrm{V}$ contents and marked depletion of incompatible elements, especially $\mathrm{Zr}$, $\mathrm{Nb}, \mathrm{Rb}$ and $\mathrm{Sr}$. These samples display distinct flat REE chondrite-normalized patterns ( $\mathrm{La} / \mathrm{Lu} 10-14)$. The REE con- 

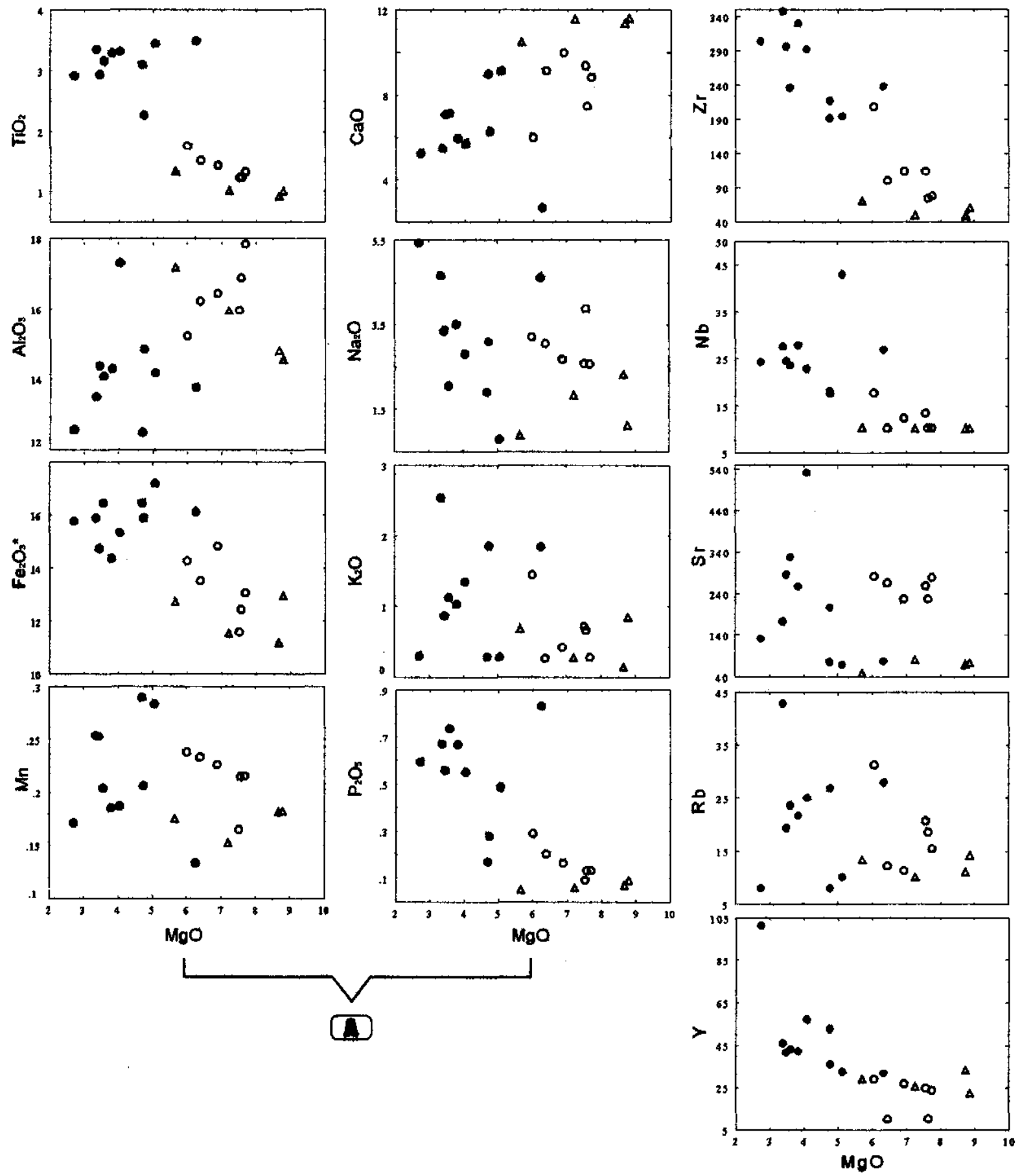

(B)

Figure 5 - Binary diagrams discriminating HTi (closed circles); LTi (open circles); and MORB-like metabasalts (triangles).

Figura 5 - Diagramas biná rios discriminando metabasitos do tipo HTi (círculos pretos); LTi (círculos brancos); e do tipo MORE (triângulos).

tents (Table 4) are the lowest, with chondrite-normalized abundances around 10 times chondrite contents (Figure $6 \mathrm{c}$ ). Marked depletion of light-REE (LaN/SmN between 0.80 and 0.97 ) and negative $\mathrm{Eu}$ anomalies (average $\mathrm{Eu} / \mathrm{Eu}^{*}=0.79$ ) are observed. In this group, a negative correlation of $\mathrm{Al}_{2} \mathrm{O}_{3}$ a and $\mathrm{MgO}$ (Figure 5) suggests that fractional crystallization of plagioclase did not substantially occur during magmatic differentiation.

The multi-elemental MORB-normalized diagram (Fig. 7c) and the tectonic discrimination diagrams (Figure 8) show that the MORB-like metabasalts have compositions more comparable with those of modern E-MORB (Humphris et al. 1985). In terms of tectonic setting, this is interpreted as magmatic contribution from Depleted Mantle source, possibly asthenospheric. For example, Figure $7 \mathrm{c}$ and also Table 5 show the remarkable similarity of these MORB-like metabasalts with an average of 5 samples of E-MORB like (REE-depleted) basalts from the Paraná volcanics (Marques 1988), which are clearly continental. 
Table 4 - Major, trace and rare-earth element contents of the MORB-like metabasic rocks from the Passos nappe, with anhydrous average and standard deviaton ( $\delta n)$. Tabela 4 - Teores de elementos maiores, traços e terras raras das rochas metabásicas do tipo MORB da nappe de Passos, com média anidra e desvio padrão (סn).

\begin{tabular}{|c|c|c|c|c|c|c|}
\hline Sample & $\begin{array}{c}\text { DEL-1. } \\
\text { 89h }\end{array}$ & $\begin{array}{c}\text { DEL-1- } \\
61\end{array}$ & $\begin{array}{c}\text { DEL-1- } \\
89 k\end{array}$ & $\begin{array}{c}\mathbf{L N}- \\
\mathbf{5 b}\end{array}$ & $\begin{array}{c}\text { Anhydrous } \\
\text { Average }\end{array}$ & $\delta_{n}$ \\
\hline \multicolumn{7}{|l|}{ wt\%: } \\
\hline $\mathrm{SiO}_{2}$ & 49.4 & 49.6 & 50.0 & 48.4 & 50.5 & 0.7 \\
\hline $\mathrm{TiO}_{2}$ & 1.3 & 1.0 & 0.92 & 1.0 & 1.1 & 0.2 \\
\hline $\mathrm{Al}_{2} \mathrm{O}_{3}$ & 16.7 & 15.7 & 14.7 & 14.4 & 15.7 & 1.1 \\
\hline $\mathrm{Fe}_{2} \mathrm{O}_{3}$ & 3.8 & 5.8 & 3.3 & 4.7 & 4.5 & 1.0 \\
\hline FeO & 7.7 & 5.0 & 7.0 & 7.3 & 6.9 & 1.1 \\
\hline $\mathrm{MnO}$ & 0.17 & 0.15 & 0.18 & 0.18 & 0.17 & 0.01 \\
\hline $\mathrm{MgO}$ & 5.5 & 7.10 & 8.6 & 8.7 & 7.6 & 1.3 \\
\hline $\mathrm{CaO}$ & 10.2 & 11.4 & 11.3 & 11,5 & 11.4 & 0.5 \\
\hline $\mathrm{Na}_{2} \mathrm{O}$ & 0.86 & 1.8 & 2.3 & 1.1 & 1.5 & 0.6 \\
\hline $\mathrm{K}_{2} \mathrm{O}$ & 0.67 & 0.27 & 0.14 & 0.84 & 0.50 & 0.29 \\
\hline $\mathrm{P}_{2} \mathrm{O}_{5}$ & 0,05 & 0.06 & 0.07 & 0.09 & 0.07 & 0.01 \\
\hline $\mathrm{H}_{2} \mathrm{O}$ & 3.35 & 1.82 & 0.93 & 0.94 & & \\
\hline $\mathrm{CO}_{2}$ & 0.25 & 0.25 & 0.25 & 0.40 & & \\
\hline LOI & 0.15 & 0.11 & 0.19 & 0.18 & & \\
\hline Total & 100.10 & 100.06 & 99.88 & 99.73 & 99.94 & \\
\hline \multicolumn{7}{|l|}{ ppm: } \\
\hline $\mathrm{Cr}$ & 342 & 411 & 411 & 68 & 315 & 145 \\
\hline $\mathrm{Ni}$ & 157 & 157 & 236 & 79 & 161 & 57 \\
\hline $\mathrm{Co}$ & 82 & 82 & 97 & 72 & 85 & 9 \\
\hline v & 290 & 260 & 270 & 260 & 277 & 15 \\
\hline $\mathbf{R b}$ & 13 & 10 & 11 & 14 & 12 & 2 \\
\hline $\mathbf{B a}$ & 81 & 72 & 72 & 90 & 81 & 8 \\
\hline Sr & 47 & 79 & 69 & 72 & 68 & 12 \\
\hline $\mathrm{Nb}$ & $<10$ & $<10$ & $<10$ & $<10$ & $<10$ & \\
\hline $\mathbf{Z r}$ & 68 & 49 & 48 & 59 & 58 & 9 \\
\hline$Y$ & 28 & 25 & 33 & 22 & 28 & 4 \\
\hline $\mathrm{Cl}$ & $<20$ & $<20$ & $<20$ & $<0$ & $<20$ & \\
\hline La & 4.81 & 3.19 & 3.08 & 2.44 & 3.47 & 0.93 \\
\hline $\mathrm{Ce}$ & 11.41 & 8.60 & 8.13 & 8.29 & 9.33 & 1.46 \\
\hline Nd & 10.66 & 6.67 & 6.90 & 5.98 & 7.75 & 1,94 \\
\hline $\mathrm{Sm}$ & 3.28 & 2.06 & 2.22 & 1.93 & 2.43 & 0.57 \\
\hline Eu & 0.92 & 0.57 & 0.65 & 0.54 & 0.69 & 0.16 \\
\hline Gd & 3.77 & 2.26 & 2.91 & 2.30 & 2.88 & 0.65 \\
\hline Dy & 4.56 & 2.84 & 3.63 & 2.92 & 3.58 & 0.73 \\
\hline Ho & 0.97 & 0.63 & 0.81 & 0.65 & 0.78 & 0.15 \\
\hline $\mathrm{Er}$ & 2.92 & 2.00 & 2.60 & 2.06 & 2,45 & 0.41 \\
\hline $\mathrm{Yb}$ & 2.71 & 1.78 & 2.26 & 1.77 & 2.18 & 0.41 \\
\hline Lu & 0.35 & 0.24 & 0.31 & 0.23 & 0.29 & 0.05 \\
\hline Mg\# & 46.85 & 55.32 & 60.59 & 57.35 & 55.03 & 5,08 \\
\hline
\end{tabular}

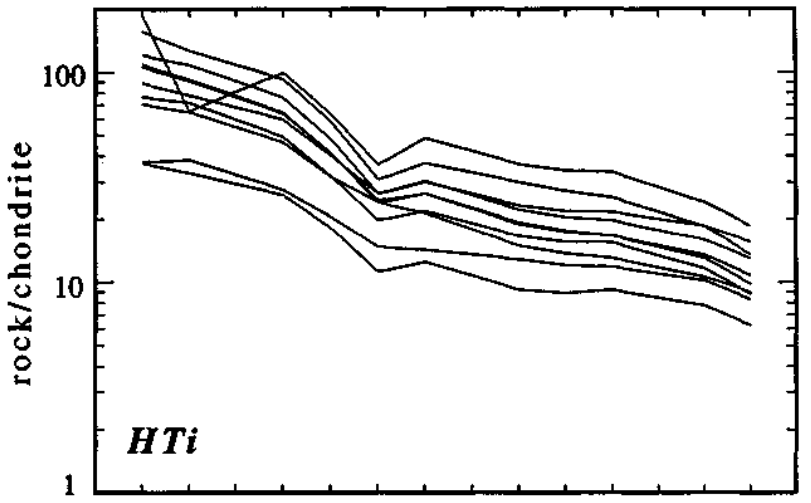

La Ce Pr Nd Sm Eu Gd Tb Dy Ho Er Tm Yb Lu

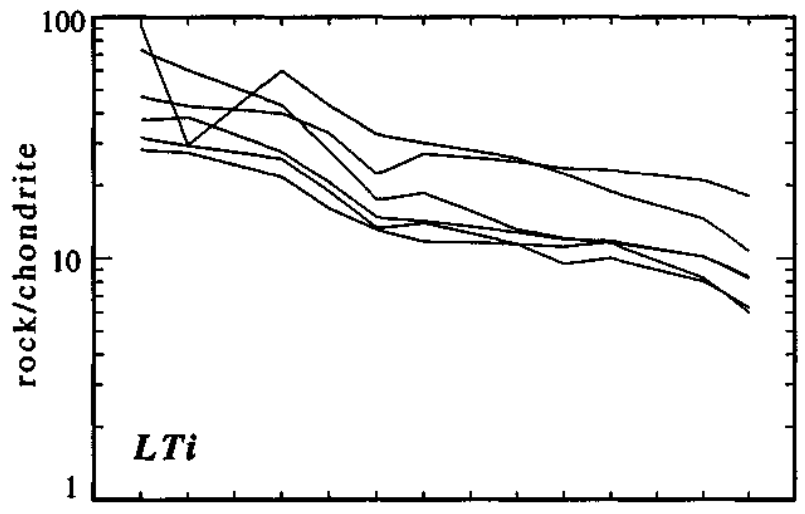

La Ce Pr Nd SmEu Gd Tb Dy Ho Er Tm Yb Lu

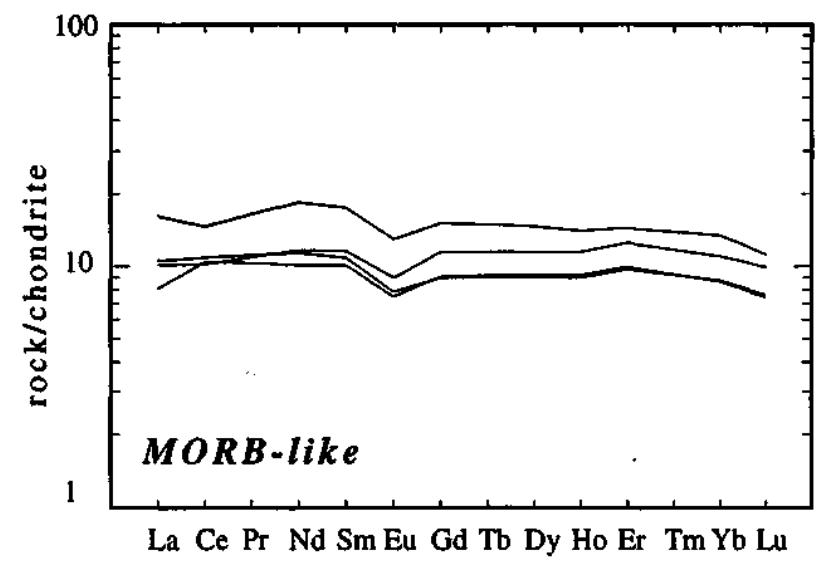

Figure 6 - Chondrite-normalized REE patterns for HTi (a), LTi (b) and MORB-like basalts (c). Normalization values from Sun (1982).

Figura 6 - Padroes de ETR para os metabasitos do tipo HTi (a), LTi (b) e MORB (c). Valores de normalização segundo Sun (1982).

DISCUSSION The distribution of samples from the 3 discriminated groups within the stratigraphic succession of the Passes nappe draws important inferences on the tectonic setting of the former post-1.8 Ga. sedimentary basin (Figure 9). The HTi group samples are concentrated in the lower section, but occur along the whole stratigraphic column; the LTi samples occur in the lower portion; and the MORB-like samples were found only in the upper portion of the stratigraphic pile. A similar distribution of typical continental and MORB-like mafic magmatism is reported by Gongalves \& Figueiredo (1992) and by Paciullo (1992) in the Andrelândia Group, a Proterozoic metasedimentary unit exposed at the southern border of the São Francisco craton, and considered 

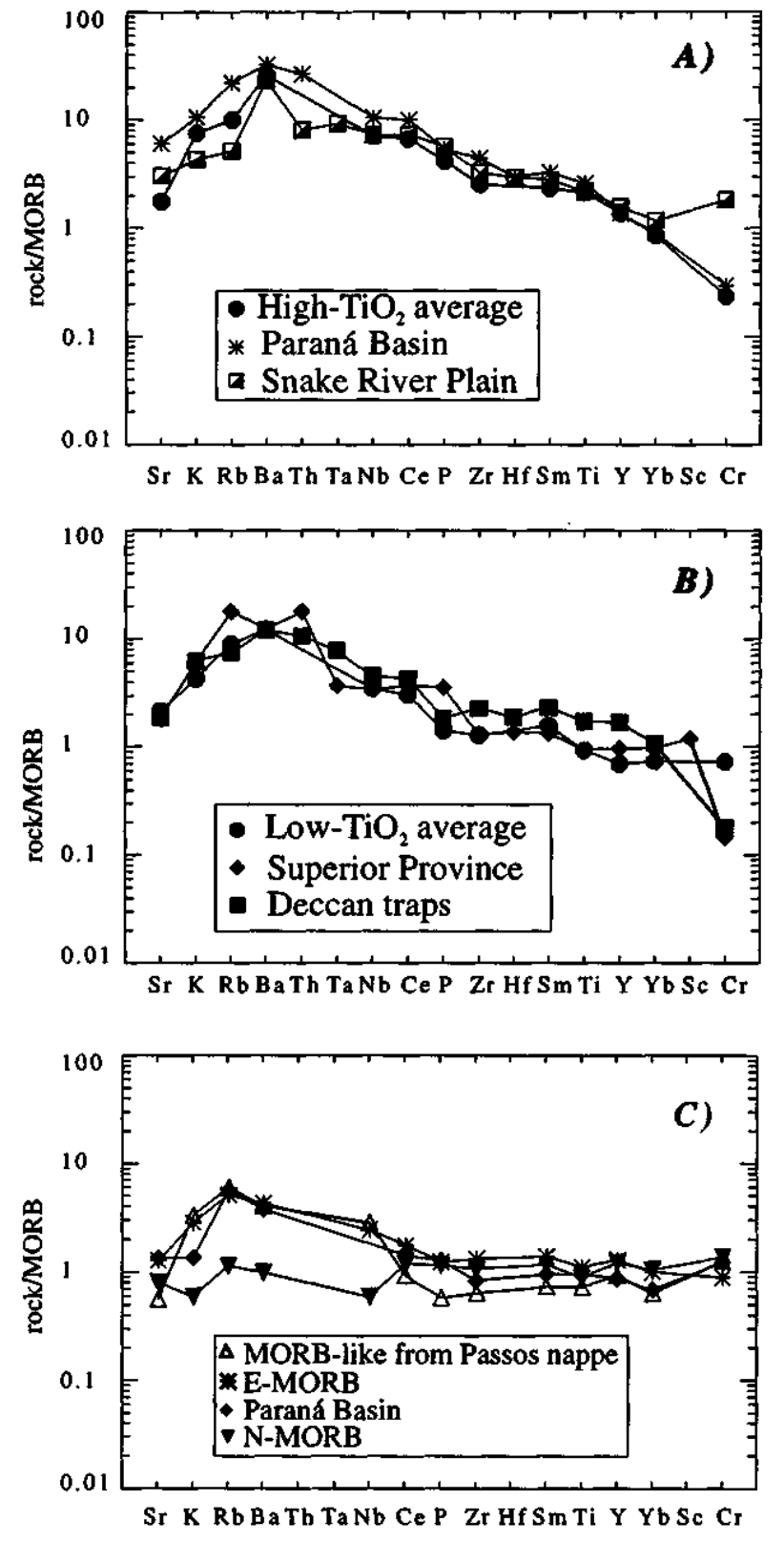

Figure 7 - MORB-normalized multi-elemental diagrams (Pearce 1983) for comparison of the averages of the three basalt types from the Passos nappe, with representative examples from other continental basalt provinces. Figura 7 - Diagramas multielementares normalizados pelo MORB (Pearce 1983), comparando as médias dos tres tipos de basaltos da Nappe de Passos com exemplos representatives de outras províncias continentais.

by Trouw et al. (1984) and Machado et al. (1983) as correlative to the Araxá Group.

The study of interactions between asthenosphere and subcontinental lithospheric mantle (SCLM) has identified several similar examples of the progressive influence of Depleted Mantle-derived magmatism, with continued lithospheric extension and plume rise, associated to CFB provinces or continental rifts. For example, Rocholl et al. (1995), based on element and isotope geochemistry, report a progressive rise and partial melting of asthenospheric Depleted Mantle source, in the Neogene-Quaternary Ross Sea rift (Antarctica), yielding MORB type magmas, as continental lithospheric extension and thinning evolved. The enriched
Table 5 - Comparison of (a) the average of the 4 MORB-like samples from the Passos nappe with (b) the average of 5 samples of basalts with similar flat REE pattern from the Parana CFB (Marques 1988).

Tabela 5 - Comparação entre: a) média de 4 amostras de basaltos tipo MORB da Nappe de Passos com b) média de 5 amostras de basaltos da Bacia do Paraná (Marques 1988) com padrões de ETR similares.

\begin{tabular}{|c|c|c|}
\hline Element & a & b \\
\hline $\mathrm{SiO}_{2}$ & 50.5 & 48.6 \\
\hline $\mathrm{TiO}_{2}$ & 1.1 & 1.4 \\
\hline $\mathrm{Al}_{2} \mathrm{O}_{3}$ & 15.7 & 14.8 \\
\hline $\mathrm{Fe}_{2} \mathrm{O}_{3} *$ & 12.1 & 11.9 \\
\hline $\mathrm{MnO}$ & 0.17 & 0.21 \\
\hline $\mathrm{MgO}$ & 7.6 & 6.6 \\
\hline $\mathrm{CaO}$ & 11.4 & 11.1 \\
\hline $\mathrm{Na}_{2} \mathrm{O}$ & 1.5 & 2.4 \\
\hline $\mathrm{K}_{2} \mathrm{O}$ & 0.5 & 0.2 \\
\hline $\mathrm{P}_{2} \mathrm{O}_{5}$ & 0.07 & 0.15 \\
\hline $\mathrm{Cr}$ & 315 & 305 \\
\hline $\mathrm{Ni}$ & 161 & 103 \\
\hline Co & 85 & 45 \\
\hline $\mathbf{R b}$ & 12 & 11 \\
\hline $\mathrm{Ba}$ & 81 & 74 \\
\hline $\mathrm{Sr}$ & 68 & 159 \\
\hline $\mathbf{Z r}$ & 58 & 74 \\
\hline $\mathbf{Y}$ & 28 & 26 \\
\hline $\mathrm{La}$ & 3.5 & 5.0 \\
\hline Ce & 9.3 & 14.1 \\
\hline Nd & 7.8 & 10.0 \\
\hline$S m$ & 2.4 & 3,1 \\
\hline Eu & 0.69 & 1.11 \\
\hline $\mathrm{Yb}$ & 2.2 & 2.3 \\
\hline Lu & 0.29 & 0.34 \\
\hline
\end{tabular}

mantle source produced the more typical continental magmatism in the initial stages of rifting. This process is more likely to occur in the more mature rifts, where adiabatic decompression and melting of depleted (and more refractory) asthenosphere requires some degree of SCLM thinning and erosion. In embryonary rifts, such as the Afar (Vidal et al. 1991), the involvement of asthenospheric Depleted Mantle is very subordinated or absent.

CONCLUSIONS Three compositional types of mafic magmatism (high- $\mathrm{TiO}_{2}$, low- $\mathrm{TiO}_{2}$ and MORB-like metabasic rocks) are identified within the Passes nappe. It is not clear from field relationships whether magmatism took place during or after sedimentation, but microstructural features indicate that their magmatic crystallization was achieved before compressional deformation.

The observed distribution of magma types favors the hypothesis of sin-depositional magmatism and indicates that the 

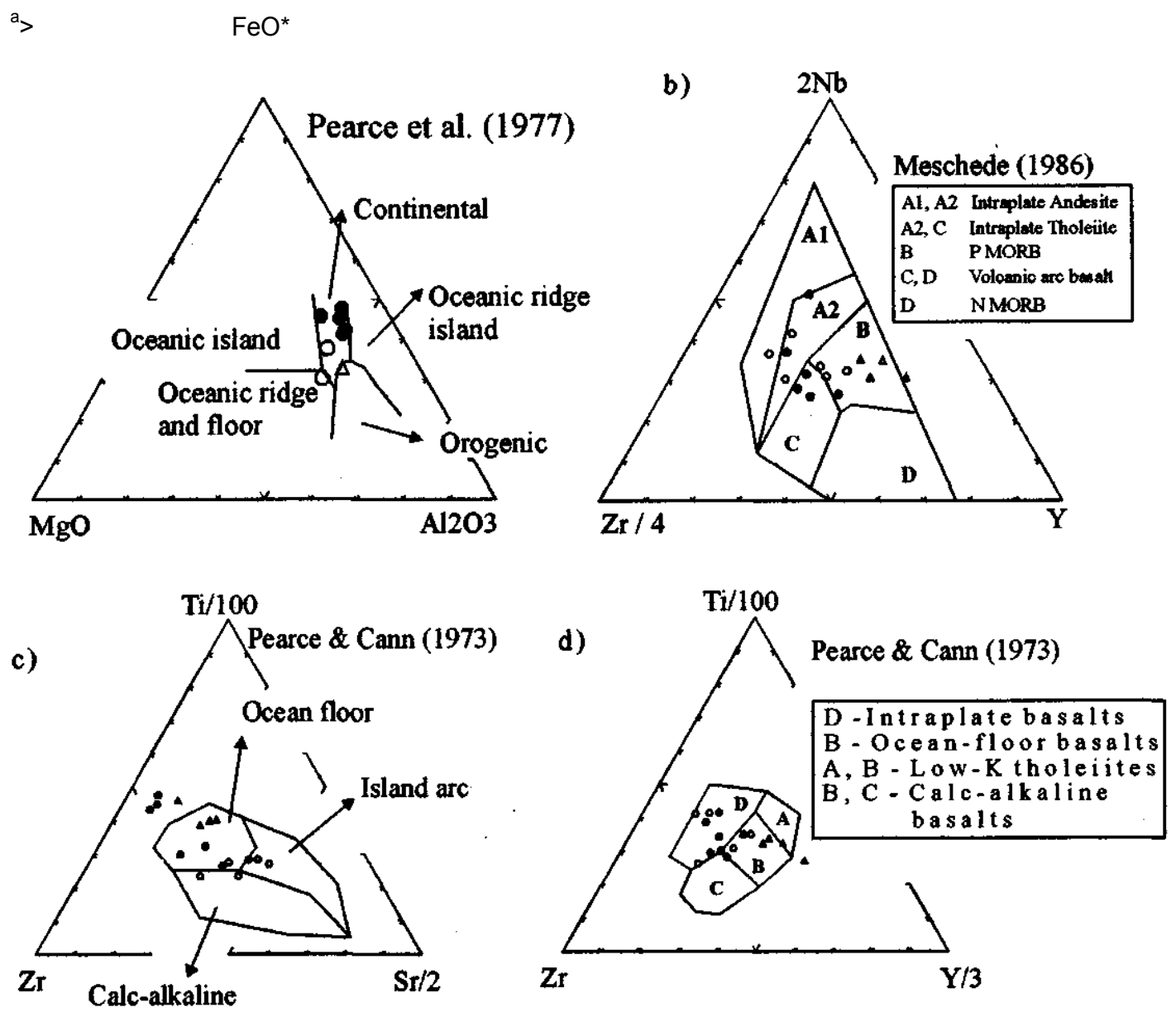

Figure 8 - Tectonic discrimination diagrams for the HTi (closed circles), LTi (open circles) and MORB-like samples (triangles). Figura 8 - Diagramas de discriminação tectônica para as amostras do tipo HTi (círculos pretos), LTi (círculos brancos) e MORB (triângulos).

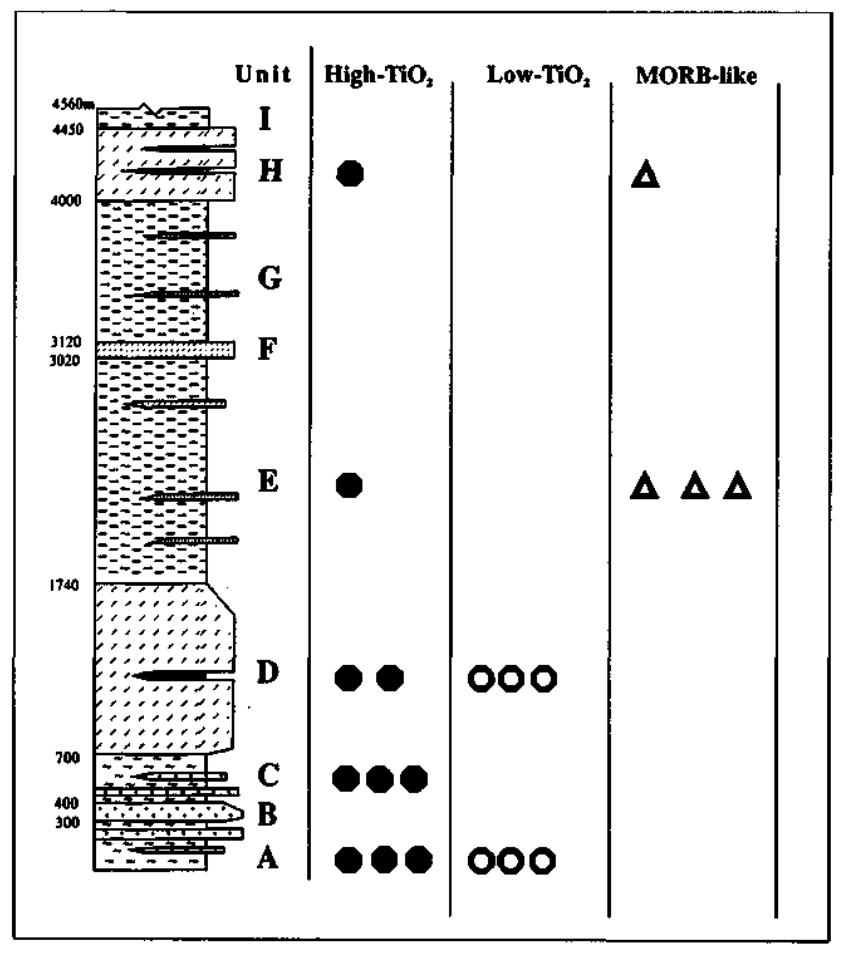

Figure 9 - Schematic distribution of the magma types along the stratigraphy of the Passos nappe.

Figura 9 - Distribuição dos tipos de metabasitos ao longo da estratigrafia da nappe de Passos. initial sedimentation of the Passos nappe metasediments (Lower Unit) was related to continental extension processes in association with CFB-type magmatism (HTi and LTi types), which continued along the rest of the preserved sedimentary record. During the sedimentation of the Upper Unit of the Passos nappe, in which relatively deeper marine conditions were probably established, MORB-like magmas were generated, probably related to more severe lithospheric extension and thinning. However, the persistence of continental magmatism in this stage is an indication that the generation of oceanic floor was not achieved during lithospheric thinning in the southern segment of the Brasilia belt. This interpretation suggests that the continental extension event responsible for the sedimentation of the Araxá Group and correlative units around the São Francisco craton could have led to varied degrees of continental fragmentation: more to the north, in Goiás state, oceanic petrotectonic associations were produced (Strieder \& Nilson 1992); more to the south, in the Passos nappe area, this event achieved only continental extension associated with deep marine environment, but without indications of true oceanic-floor generation.

Acknowledgements Professor Mario C. H. de Figueiredo is kindly acknowledged for first encouraging this study and for introducing CMV to lithogeochemical studies. Analyses were supported by FINEP-IG/USP funds (43.89.0526.00). The comments and suggestions made by two anonymous reviewers contributed to significant improvement of the manuscript. 


\section{REFERENCES}

ALMEIDA, F.F.M.; HASUI, Y.; BRITO NEVES, B.B. 1976. The upper Precambrian of South America. Boletim do IG Institute de Geociencias, USP, v. 7:45-80

BARBOSA, O.; BRAUN, O.P.G.; DYER, R.C.; CUNHA, C.A.B.R. 1970 Geologia da região do Triângulo Mineiro. Departamento National de Produção Mineral - Boletim da Divisão de Fomento da Produção Mineral, $136,140 \mathrm{p}$

BELLIENI, G.; CHIARAMMONTI, P.C.; MARQUES, L.S.; MELFI, A.J.; PICIRILLO, E.M.; NARDY, A.J.R.; ROISENBERG, A., 1986. High and low- $\mathrm{TiO}_{2}$ flood basalts from the Paraná plateau (Brazil): petrology and geochemical aspects bearing on their mantle origin. Neues Jahrbuch Mineral. Abb. 150(3):273-306.

CONDIE, C.K.; DOBROW, D.J.; CARD, K.D. 1987. Geochemistry of Precambrian mafic dykes from the southern Superior Province of the Canadian Shield. In: Halls, H.C. \& Fahrig, W.F. (eds.) Mafic dyke swarms. Geological Association of Canada Special Paper 34:95-108.

CORREIA, C.T. 1986. Geologia da Folha Cassia, MG, epetrologia de seus anfibolitos. Unpublished Master Thesis, Institute de Geociências, Universidade de São Paulo, $123 \mathrm{p}$.

CORREIA, C.T.; KAWASHITA, K.; GIRARDI, V.A.V. 1982. Datacões $\mathrm{Rb}-\mathrm{Sr}$ e K-Ar em rochas metamórficas da região de Cássia (MǴ) e considerações sobre seu significado geológico. Anais da Academia Brasileira de Ciências, 54(4): 673-677.

COX, K.G. 1978. Flood basalts, subduction and the break-up of Gondwanaland. Nature, 274:47-49.

DE LA ROCHE, H.; LETERRIER, J.; GRANDCLAUDE, P.; MARCHAL, M. 1980. A classification of volcanic and plutonic rocks using R1R2diagram and major-element analyses - its relationships with current nomenclature. Chemical Geology, 29(3/4): 183-210.

GONCALVES, M.L. \& FIGUEIREDO, M.C.H. 1992. Geoquímica dos anfibolitos de Santana do Garambéu (MG): implicaçỗes sobre a evolução do Grupo Andrelândia. Geochimica Brasiliensis, 6(2): 127 140.

HUMPHRIS, S.E.; THOMPSON, G. SHILLING, J.G.; KINGSLEY, R.A. 1985. Petrological and geochemical variations along the Mid-Atlantic Ridge between $46^{\circ} \mathrm{S}$ and $32^{\circ} \mathrm{S}$ : influence of the Tristan da Cunha mantle Plume. Geochimica et Cosmochimica Acta 49:1445-1464.

IRVINE, T.N. \& BARAGAR, W.R.A. 1971. A guide to the chemical classification of the common volcanic rocks. Canadian Journal of Earth Sciences, 8:523-548.

LE BAS, J.J.; LE MAITRE, R.W.; STRECKEISEN, A.; ZANETTIN, B. 1986. A chemical classification of volcanic rocks based on the total alkali silica (TAS) diagram. Australian Journal of Earth Science, 31(2):243-255.

MACHADO, L.; RIBEIRO, M.; GONZALEZ, S.R.; SCHENINI, C.A.; SANTOS NETO, A.; PALMEIRA, R.C.; PIRES, J.L.; TEIXEIRA, W.: CASTRO, H.E.F. 1983. Geologia da folha Rio de Janeiro, escala 1:1.000.000, mapa e texto explicativo. RADAMBrasil - Ministério das Minas e Energia, Rio de Janeiro, $780 \mathrm{p}$.

MARINI, O.J.; FUCK, R.A.; DARDENNE, M.A.; DANNI, J.C.M. 1984 Provínca Tocantins. Setores Central e Sudeste. In: ALMEIDA, F.F.M. \& HASUI, Y. eds. O Pré-cambriano do Brasil. São Paulo, Edgar Blucher, p. 205-264.

MARQUES, L.S. 1988. Caracterização geoquimica das rochas vulcânicas da Bacia do Paraná: implicates petrogenéticas. Tese de Doutorado, Institute Astronômico e Geofísico - Universidade de São Paulo, 175 p.

MESCHEDE M. 1986. A method of discriminating between different types of mid-ocean ridge basalts and continental tholeiites with a Nb-Zr-Y diagram. Chemical Geology, 56: 207-218.

MIYASHIRO, A. 1975. Classification, characteristics and origin of ophiolites. Journal of Petrology 83(2):249-281.

MULLEN, E.D. 1983. $\mathrm{MnO} / \mathrm{TiO}_{2} / \mathrm{P}_{2} \mathrm{O}_{5}$ : a minor element discriminant for basaltic rocks of oceanic environments and its implications for petrogenesis. Earth and Planetary Science Letters, 274:53-62.

PACIULLO, F.V.P. 1992. Orto-anfibolitos no ciclo deposicional Andrelândia - assinatura química e ambiente tectônico. In: Congresso Brasileiro de Geologia, 37, São Paulo, 1992, Anais...São Paulo, $\mathrm{SBG} / \mathrm{SP}$, vol 2:28-30

PEARCE, J.A. 1983. Role of the sub-continental lithosphere in magma genesis at active continental margins. In: C.J. Hawkesworth and M.J. Norry (eds.), Continental Basalts and Mantle Xenoliths. Shiva, Nantwich, pp. 230-249.
PEARCE J.A. \& CANN J. 1973. Tectonic setting of basic volcanic rocks determined using trace element analysis. Earth and Planetary Science Letters, 19: 290-300.

PEARCE, T.H.; GORMAN, B.E.; BIRKETT, T.C. 1977. The relationship between major element chemistry and tectonic environment of basic and intermediate volcanic rocks. Earth and Planetary Science Letters 36(1):121-132.

PIMENTEL, M.M. \& FUCK, R.A. 1992. Neoproterozoic crustal accretion in central Brazil. Geology, v. 20: 375-379.

PIMENTEL, M.M. \& FUCK, R.A. 1994. Geocronologia Rb-Sr da porcão sudoeste do Maciço de Goiás. Revista Brasileira de Geociências 24(2): 104-111.

ROCHOLL, A.; STEIN, M.; MOLZAHN, M.; HART, S.R.; WORNER, G. 1995. Geochemical evolution of rift magmas by progressive tapping of a stratified mantle source beneath the Ross Sea Rift, Northern Victoria Land, Antarctica. Earth and Planetary Science Letters, 131:207-224.

SCHMIDT, W. 1983. Die Geologie der Araxa Gruppe in Sudwest Minas Gerais, Brasilien unter besonderer Berucksichtigung des Grunsteingurtels van Fortaleza de Minas. Unpublished Thesis, Albert-Ludwigs Universitat, Freiburg, DFR, 134 p.

SIMÕES, L.S .A. 1995. Evolução tectonometamórfica da Nappe de Passos, sudoeste de Minas Gerais. Tese de Doutorado, Institute de Geociências, Universidade de São Paulo, 149 p.

SIMÕES, L. S. A. \& VALERIANO, C. M. 1990. Porção meridional daFaixa de Dobramentos Brasília: estágio atual do conhecimento e problemas de correlacão tectono-estratigráfica. In: Congresso Brasileiro de Geologia., 36, Natal, 1990, Anais... Natal, SBG, 6:2564-2575.

STRIEDER, A.J. \& NILSON, A.A. 1992. Melange ofiolítica nos metassedimentos Araxá de Abadiânia (GO) e implicacões tectônicas regionais. Revista Brasileira de Geociências 22(2):204-215.

SUN, S.S. 1982. Chemical composition and origin of the Earth's primitive mantle. Geochimica et Cosmochimica Acta 46:179-192.

SZABO, G. A. J.; ROIG, H.L.; SCHRANK, A. \& CHOUDHURI, A. 1993. Duas faixas lito-estruturais distintas e o limite das Províncias Tocantins e Mantiqueira, entre Alpinóplis e Nova Resende. In: Simpósio sobre o Cráton do São Francisco e suas faixas marginais, 2, Salvador, 1993, Anais... Salvador, SBG, pp. 274-274.

TEIXEIRA, N.A. 1996. Assoalho oceânico no complexo ultramático do Quatipuru (PA). Implicates geotectônicas para a Faixa Araguaia. In: Congresso Brasileiro de Geologia, 39, Salvador, 1996, Anais...Salvador, SBG/BA-SE, 6:117-120.

TEIXEIRA, N.A. \& DANNI, J.C.M. 1978. Contribuição a estratigrafia do Grupo Araxá na região de Passos (MG). In: Congresso Brasileiro de Geologia, 30, Recife, 1978, Anais...Recife, SBG, 2:700-710.

THOMPSON, R.N.; MORRISON, M.A.; DICKIN, A.P.; HENDRY, G.L. 1983. Continental flood basalts... arachnids rule OK? In: Hawkesworth, C.J. \& Norry, M.J. (eds.) Continental basalts and mantle xenoliths. Nantwich, Shiva, p. 158-185.

TROUW, R.A.J.; RIBEIRO, A.; PACIULLO, F.V.P.; HEILBRON, M. 1984. Os Grupos São João del Rei e Andrelândia, interpretados como a continuação lateral dos grupos Canastra e Araxá In: Congresso Brasileiro de Geologia, 33, Rio de Janeiro, 1984, Anais... Rio de Janeiro, SBG, vol. 7: 3227-3240.

VALERIANO, C. M. 1992. Evolucão tectônica da extremidade meridional da Faixa Brasília região da Represa de Fumas sudoeste de Minas Gerais. Tese de Doutorado, Institute de Geociências -Universidade de São Paulo, 192 p.

VIDAL, P.: DENIEL, C · VELLUTINI, P.J.; PIGUET, P.; COULON, C. VINCENT, J.; AUDIN, J. 1991. Changes of mantle sources in the course of a rift evolution: the Afar case. Geophysical Research Letters, 18(10): 1913-1916.

Manuscrito A884

Recebido em 15 de novemro de 1996 Revisao dos autores em 15 de abril de 1997 Revisao aceita em 15 de junho de 1997 